\title{
Psychosocial mediators of change in physical activity in the Welsh national exercise referral scheme: secondary analysis of a randomised controlled trial
}

Hannah J Littlecott ${ }^{1{ }^{*} \dagger}$, Graham F Moore ${ }^{1 *^{*}}$, Laurence Moore ${ }^{2}$ and Simon Murphy ${ }^{1}$

\begin{abstract}
Objective: While an increasing number of randomised controlled trials report impacts of exercise referral schemes (ERS) on physical activity, few have investigated the mechanisms through which increases in physical activity are produced. This study examines whether a National Exercise Referral Scheme (NERS) in Wales is associated with improvements in autonomous motivation, self-efficacy and social support, and whether change in physical activity is mediated by change in these psychosocial processes.
\end{abstract}

Methods: A pragmatic randomised controlled trial of NERS across 12 LHBs in Wales. Questionnaires measured demographic data and physical activity at baseline. Participants $(N=2160)$ with depression, anxiety or CHD risk factors were referred by health professionals and randomly assigned to control or intervention. At six months psychological process measures were collected by questionnaire. At 12 months physical activity was assessed by 7 Day PAR telephone interview. Regressions tested intervention effects on psychosocial variables, physical activity before and after adjusting for mediators and socio demographic patterning.

Results: Significant intervention effects were found for autonomous motivation and social support for exercise at 6 months. No intervention effect was observed for self-efficacy. The data are consistent with a hypothesis of partial mediation of the intervention effect by autonomous motivation. Analysis of moderators showed significant improvements in relative autonomy in all subgroups. The greatest improvements in autonomous motivation were observed among patients who were least active at baseline.

Discussion: The present study offered key insights into psychosocial processes of change in an exercise referral scheme, with effects on physical activity mediated by autonomous motivation. Findings support the use of self-determination theory as a framework for ERS. Further research is required to explain socio-demographic patterning in responses to ERS, with changes in motivation occurring among all sub-groups of participants, though not always leading to higher adherence or behavioural change. This highlights the importance of socio-ecological approaches to developing and evaluating behaviour change interventions, which consider factors beyond the individual, including conditions in which improved motivation does or does not produce behavioural change.

Trial register number: ISRCTN47680448

\footnotetext{
*Correspondence: LittlecottH@cardiff.ac.uk; MooreG@cardiff.ac.uk

'Equal contributors

'DECIPHer, School of Social Sciences, Cardiff University, 1-3 Museum Place,

Cardiff, Wales, UK

Full list of author information is available at the end of the article
}

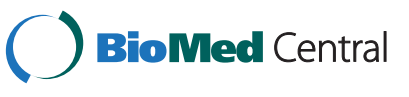

(c) 2014 Littlecott et al.; licensee BioMed Central Ltd. This is an Open Access article distributed under the terms of the Creative Commons Attribution License (http://creativecommons.org/licenses/by/2.0), which permits unrestricted use, distribution, and reproduction in any medium, provided the original work is properly credited. The Creative Commons Public Domain Dedication waiver (http://creativecommons.org/publicdomain/zero/1.0/) applies to the data made available in this article, unless otherwise stated. 


\section{Introduction}

While physical activity is comparable to smoking and diet in terms of its influence on chronic disease outcomes, most UK adults are insufficiently active [1]. Recent decades have seen growing interest in interventions to increase physical activity [2], with exercise referral schemes (ERS) one approach to increasing activity among at-risk groups. Typically these involve health professional referral to a leisure facility, agreement of an exercise programme with an instructor, and discounted access to leisure facilities for 10-12 weeks. While proliferating rapidly in recent years [3], randomised controlled trials (RCTs) and observational studies have indicated disappointing long-term impacts of ERS on physical activity [4-6]. However, while grouped under the term ERS, schemes are highly variable in their design and delivery [7], reflecting varying assumptions regarding how best to affect change.

The National Exercise Referral Scheme in Wales was implemented from 2007, and evaluated using a pragmatic randomised trial [8], which maximised external validity through evaluating a real world intervention with minimal interference from the evaluation team in how the scheme was implemented. Trial findings indicated that the intervention was associated with significant improvements in physical activity for patients referred with coronary heart disease risk factors (though not for patients referred for mental health reasons) [9]. However, in evaluating complex interventions such as exercise referral schemes, there is a need to move beyond evaluating overall effects, and towards understanding the theoretical assumptions being made by the intervention, and the mechanisms through which change is achieved in context, through mixedmethod process evaluation [10].

The NERS evaluation included a comprehensive process evaluation, which aimed to articulate its theories of change, and to evaluate its implementation and causal mechanisms. While NERS was not based on an explicit theory of behaviour change, all interventions can be described as 'theories incarnate' [11], in that they reflect causal assumptions about how change is to be produced. Hence, the NERS process evaluation began with the development of a logic model through discussions between programme developers and the evaluation team, which set out the causal assumptions of the intervention. Subsequently, intervention fidelity and dose were closely monitored, indicating some deviations from the intended programme, with for example, motivational interviewing and goal setting not fully delivered $[12,13]$. Qualitative exploration of professionals' and patients' perceptions then provided key insights into the mechanisms through which the intervention worked in practice. For example, patients highlighted the role of patient only classes in supporting their sense of competence and relatedness to one another, through provision of realistic exemplars for social comparison [13]. Roles of exercise professionals in supporting patients' confidence in using unfamiliar machinery, and exercising safely within the limits of their conditions were highlighted by patients and professionals [12,13].

While inductive qualitative methods are crucial in understanding how complex interventions facilitate change, quantitatively testing hypothesised mediators of effect is also central to building theory $[13,14]$. While like NERS, most ERS are not explicitly based on a single behaviour change theory, some efforts have been made to apply psychological theory and quantitatively test hypothesised mechanisms of impact. Most commonly, such studies have tested change in constructs from self-efficacy theory [15] or self-determination theory [16]. Self-determination theory (SDT) argues that stable change is most likely where individuals have high levels of autonomous motivation (i.e. where they find exercise intrinsically enjoyable, or link it to personally valued outcomes). In turn, development of more autonomous motivation is likely in contexts which support psychological needs for autonomy, competence and relatedness. Associations have been consistently observed between levels of autonomous motivation and physical activity [17]. For example, a systematic review of 66 empirical studies found a consistent association between autonomous motivation and physical activity [18].

In one observational study examining change in SDT constructs within an ERS, Edmunds et al. [19] found that patients for whom motivations became more internalised were more likely to adhere than those for whom participation remained externally motivated. Likewise, Morton et al. [20] found that patients who completed an ERS demonstrated greater self-determined motivation than those who did not. Markland and Tobin [21] argue that as patients typically enter ERS on the advice of a health professional, behaviour change is initially externally motivated, with ERS needing to promote internalisation of motivation. Their survey of 133 female completers of a 10 week scheme, reported higher identified motivation (i.e. acting due to a sense of the behaviour as personally important) amongst patients who perceived that the professional was supportive of autonomy and competence [21], as well as amongst those who reported higher levels of social assimilation into the exercise environment and higher levels of relatedness to others within that environment. Moreover, studies have shown that general practitioners who implement an autonomy supportive style can affect patients' autonomous motivation [22]. One physical activity counselling trial has found that brief autonomy-supportive counselling by a physician, followed by counselling from a physical activity counsellor, significantly increased autonomous motivation at six weeks, which then predicted physical activity at 13 weeks [17]. 
Self-efficacy theory argues that behaviour change is likely where seen as an effective means of achieving desired outcomes, and as within the individual's capabilities [15]. Studies have reported associations of self-efficacy with physical activity among participants with ages ranging from 18 to 92 [23]. In a study examining effects of an ERS on self-efficacy, Jones et al. [24] found that self-efficacy improved amongst completers of a 12-week gym-based programme, though worsened among non-adherers, indicating potential beneficial impacts, though adverse effects of trying but failing to change. Their high drop-out rate makes it difficult to generalise from these results. Mediation of self-efficacy on physical activity was examined in a longitudinal study of exercise in cardiac rehabilitation patients, which showed evidence of mediation of the relationship between exercise intentions at baseline and physical activity at two and four months by self-efficacy [25].

Social support has been shown to be an important correlate of physical activity. A meta-analysis reported social influence in the form of family support and attitudes towards exercise, task cohesion and adherence, important others' attitudes about exercise and family compliance and support to have a moderate to large effect on participation in exercise [26]. In a review of exercise referral schemes, qualitative assessment of participants' reasons for adherence showed that social benefits were gained from participation, whilst poor social support was found to be a reason for non-adherence [4]. In qualitative interviews conducted within the NERS process evaluation [13], patients emphasised that referral to an ERS increased the extent to which family members supported their taking time out of family commitments to exercise.

In evaluating the effectiveness of interventions such as ERS, RCTs are usually considered to be the 'gold standard'. However, while an increasing number of RCTs have examined impacts of ERS on physical activity, attempts to unpick psychosocial processes of change have often relied upon less robust observational methods. Few studies have to date examined the extent to which changes in psychosocial processes predict longer-term change in physical activity behaviour.

The main aim of this paper is to test the following primary hypotheses:

- Referral to NERS was associated with improvements in autonomous motivation, self-efficacy for exercise and perceived social support for exercise at 6 month follow-up.

- Impacts of NERS on physical activity at 12 months were mediated by change in these psychosocial processes.

In addition, the paper will examine patterning in changes in psychosocial mediators of change by sociodemographic factors.

\section{Methods}

The national exercise referral scheme

NERS comprised health professional referral to a local authority leisure centre, a one-to-one consultation with an exercise professional, a 16 week programme of supervised group based activity, with follow ups from the professional at 4 and 16 weeks, then again at 8 and 12 months. The intervention, as intended and as delivered in practice, is described in detail elsewhere [13].

\section{Study design and procedures}

The study was a pragmatic randomised controlled trial. Patients were recruited from 12 local health boards (LHBs) across Wales using opportunistic referral by a range of health professionals. At baseline, participants completed a short postal questionnaire assessing age, gender, marital status, ethnicity, employment status, education, number in household and the General Practice Physical Activity Questionnaire (GPPAQ) [27], which provides a patient activity index to indicate whether the respondent is inactive, moderately inactive, moderately active, or active. Participant postcodes were also used to calculate a measure of deprivation based on the Welsh Index of Multiple Deprivation (WIMD). Those who consented and returned baseline questionnaires were randomly assigned to the intervention or control trial arm using a random number generator, with gender and LHB as stratification variables. Randomisation of referrals occurred every 2 weeks, with treatment allocation blind to condition and remote from participants and practitioners. Control participants were offered priority access to NERS at 12 months, following a health check. Six month psychosocial process measures were collected by postal questionnaire. While the intended intervention duration was 16 weeks, it typically took around 6 weeks for patients to attend their first session after referral, while the average time from first appointment to scheme exit was 19 weeks [13]. Hence, 6 month follow-up typically coincided with the end of the intervention period. The research team were responsible for distributing, collecting and processing postal questionnaires, with non-responders sent a repeat mailing two weeks after the first. At 12 months, physical activity was assessed by telephone interview. A specialist health research team were employed to conduct telephone interviews using a standardised protocol [28]. Detailed descriptions of trial methodology are reported in the protocol paper [8]. The Thames Valley Multi-centre Research Ethics Committee (MREC) approved the evaluation of the Welsh NERS on 8 Feb 2007 (Ref: 06/MRE12/85). The conduct of the study conformed to the principles embodied in the Declaration of Helsinki. Written informed consent was obtained from all participants. 


\section{Psychosocial process measures Autonomous motivation}

Autonomous motivation for exercise was measured using the Behavioural Regulations in Exercise Questionnaire [29]. The measure includes 19 items which assess motivation on a continuum from less to more autonomous. Subscales of the measure are 'amotivation' (e.g. I don't see the point in exercising), 'external regulation' (e.g. I feel guilty when I don't exercise), 'introjected regulation' (e.g. I feel under pressure from my friends and family to exercise'), 'identified regulation' (e.g. 'It's important to me to exercise regularly') and 'intrinsic motivation' (e.g. 'I enjoy my exercise sessions). All 5 subscales demonstrated good internal consistency (alphas 0.74 to 0.91). A 'relative autonomy index' (RAI) is constructed by multiplying mean item scores for each subscale by a weighting value, and summing them. Weighting values are -3 for amotivation, -2 for external, -1 for introjected, 2 for identified and 3 for intrinsic regulation. An alternative method of deriving a composite measure for autonomous motivation is to average the autonomous motivation scales (identified and intrinsic regulation). As a sensitivity analysis, we ran our analyses using both measures, which produced very similar results. Hence, we report data only relating to the RAI. Exploratory factor analysis indicated that the original factor structure was perfectly replicated, with the exception that intrinsic and identified regulation items loaded onto a single factor. This measure has been used previously in a study to assess changes in autonomous motivation within patients participating in an exercise on referral scheme [21].

\section{Self-efficacy for exercise}

A revised version of the original Self-efficacy for Exercise Behaviours [30] questionnaire was also administered at 6 months. This measure includes subscales for 'sticking to it', which includes questions regarding overcoming barriers to exercise, such as demands from work and family, and 'making time for exercise', which includes questions about getting up early to exercise at the weekend and setting aside time to exercise. Both demonstrated good internal consistency (alpha $=0.93$ and 0.82). However, exploratory factor analysis indicated that the 4 items from the 'time' subscale loaded onto 3 separate factors. Hence, analysis focused on the first subscale. Furthermore only 5 of the 9 items related to 'sticking to it' loaded onto the first factor (along with 2 items from the 'time' subscale). This factor was constructed both as per Sallis and colleagues' coding and as per the factor analysis. Both variables were almost perfectly correlated $(r=0.95)$. Therefore, to facilitate comparability with other studies using the measure, analyses used Sallis and colleagues' original coding.

\section{Social support for exercise}

Sallis and colleagues' Social Support for Exercise Behaviours questionnaire was administered at 6 month follow up [31].
It included subscales relating to social support from family and friends, in terms of participation and involvement (e.g. asking patients to indicate how often friends/family 'exercised with me'), and rewards and punishment (used only for family). For friends and family, the first subscale demonstrated good reliability (alpha $=0.90$ ) However, the 'rewards and punishment' subscale demonstrated poor reliability (alpha $=0.41$ ) and was not used. Furthermore, an exploratory factor analysis indicated that the first factor (participation and involvement) divided into 3 sub-scales. These were 'exercising together' (e.g. exercised with me), 'verbal support for exercise' (e.g. gave me helpful reminders to exercise) and 'planning for exercise (e.g. 'helped plan activities around my exercise'). Intervention effects on social support using the participation and involvement scale as defined by Sallis and colleagues, and these emerging sub-scales (alphas 0.70 to 0.87 ) were examined.

\section{2 month physical activity}

Total minutes of weekly exercise of a moderate intensity (defined as how the participant feels when walking at a normal pace) or greater was assessed using the seven day physical activity recall (7D-PAR) [32]. This interview-based measure has been validated in community and experimental studies [32] and used in several randomised controlled trials to evaluate the effectiveness of physical activity promotion strategies [33,34], including several high-quality exercise referral scheme evaluations [35-38].

\section{Analysis}

To examine Hypothesis 1, the effects of the intervention on psychosocial process variables were assessed using linear regression for autonomous motivation (with initial skewness corrected using a square root transformation), and ordinal regression models for tertile scores of all remaining variables whose skewness could not be corrected. Models adjusted for baseline physical activity, health board area, gender, age and reason for referral. Subsequently, for psychosocial process variables which were shown to have been significantly impacted by the intervention, their significance as a mediator of change in physical activity is assessed in 2 two ways. First, Baron and Kenny's causal steps approach is used [39]. Regression models estimate i) the effects of the intervention on physical activity before adjustment for the mediator and ii) the effects of the mediator on physical activity after adjustment for the intervention term and iii) effects of the intervention on physical activity after adjustment for the mediator. Where the mediator is shown to be significantly associated with the outcome, and reduces the effect of the intervention, a hypothesis of mediation is supported. Second, the product of coefficients [40] is calculated using the logistic regression mediation plug-in in Stata 11, 
with the proportion of total effect explained by indirect effects presented.

As in the main trial, due to negative skew, the physical activity measure was divided into quintiles $(0=0$ minutes per week, $1=1-149,2=150-299,3=300-599,4=600+$ ) and subjected to ordinal logistic regression. As a sensitivity analysis, effects on physical activity were also tested using negative binomial regression models, which gave comparable results. To maintain direct comparability with the original analyses of trial main effects, we report only results from the ordinal analyses. Given that the main trial [9] indicated effects on physical activity only for the subgroup of patients referred for CHD risk,and that we were interested in understanding the processes underlying this significant effect, mediational analyses were limited to this subsample. Finally, for variables shown to mediate intervention effects on physical activity, socio-demographic patterning in change in these processes was evaluated i) using separate regression models for each subgroup, for gender, reason for referral, age, baseline PA level, level of deprivation, trial status and adherence level and ii) using whole group regression models with intervention"moderator interaction terms.

\section{Results}

\section{Retention}

At baseline, 1080 participants were randomised to each trial arm. Of these patients, 581 (53.8\%) intervention patients and $583(53.9 \%)$ control patients completed at least one of the 6 month psychosocial process measures. Of these, $440(40.7 \%)$ intervention patients and 466 (43.1\%) control patients completed 12 month follow up measures. Drop out was not significantly different by trial arm. Table 1 shows the proportion of the sample for whom data are available on at least one 6 month measure, and 12 month physical activity. Complete data were significantly more likely to be available for older patients, non-mental health patients, those from less deprived groups, and those who completed NERS.

\section{Intervention effects on psychosocial process variables}

Table 2 shows effects of the intervention on each of the psychosocial process measures at 6 month follow up. For the autonomous motivation subscales, there is evidence of significant reduction in amotivation, significant improvements in introjected, identified and intrinsic motivation, and no change in external motivation. Intervention effects appear stronger for more self-determined forms of motivation (e.g. intrinsic vs introjected). Significant improvements are also observed for the composite autonomous motivation scores derived from these 5 subscales. There is no evidence of an intervention effect on self-efficacy for exercise. There is evidence of significantly greater social support for exercise from friends and family among patients referred to NERS compared to controls. Analysis of the sub-scales emerging from factor analysis indicated that for both family and friends, the strongest effects were observed for 'verbal support' for exercise. For neither family nor friends was referral to NERS associated with reported increases in family members and friends exercising with the patient, while for family only, there were significant reported increases in family support for planning for exercise.

\section{Mediation of change in physical activity among patients referred for CHD risk factors}

Numbers and percentages of participants within each physical activity quintile, among participants who provided at least one psychosocial process measure, are presented in Table 3. For neither intervention nor control groups did patients who did or did not complete psychosocial process measures differ in terms of physical activity at follow-up. Table 4 presents the effects of the intervention on physical activity before and after adjusting for psychosocial mediator variables. Self-efficacy is excluded from this analysis due to a lack of intervention effect. For autonomous motivation, after entry of the mediator, the intervention effect on physical activity is reduced from an OR of 1.39 to 1.27 , becoming non-significant. For family social support, the odds ratio for intervention effect is reduced from 1.46 to 1.38, remaining significant. For social support from friends, the intervention effect is reduced only very slightly, from 1.40 to 1.37 , remaining significant. Notably, the intervention effect estimate is higher for the sub-sample of participants who completed social support measures, declining only to a level comparable to the unadjusted effect estimates for autonomous motivation. Hence, there is support for a hypothesis of partial mediation of the intervention effect by autonomous motivation, though limited evidence of a mediating effect of social support. Where all 3 psychosocial process measures and the intervention term are entered together, only autonomous motivation remains a significant predictor of physical activity ( $\mathrm{OR}=1.34,95 \%$ $\mathrm{CI}=1.14$ to 1.58$)$ with the intervention effect reduced to $1.22(95 \% \mathrm{CI}=0.90$ to 1.64$)$. Odds ratios for family and friend social support are reduced to 1.14 (0.96 to 1.36) and 1.16 (0.93 to 1.46) respectively. Product of coefficients tests indicate that of the total intervention effect on physical activity, $44.0 \%(\mathrm{p}<0.01)$ is explained by autonomous motivation, $15.5 \%(\mathrm{p}=0.07)$ by social support from family and $3.1 \%(\mathrm{p}=0.51)$ by social support from friends. Hence, there is support for a hypothesis of mediation by autonomous motivation, smaller but near significant mediation by family social support, though no support for a hypothesis of mediation by social support from friends.

\section{Moderators of change in relative autonomy}

Table 5 indicates that significant improvements in relative autonomy occurred in all subgroups, with the exception 
Table 1 Number (and percentage) of participants providing complete data by subgroup

\begin{tabular}{|c|c|c|c|c|}
\hline & & & Control & Intervention \\
\hline \multirow[t]{2}{*}{ Gender } & Male & $316(42.4)$ & $152(40.8)$ & $164(44.1)$ \\
\hline & Female & $590(41.7)$ & $314(44.4)$ & $276(39.0)$ \\
\hline \multirow[t]{3}{*}{ Age } & $16-44$ & $191(29.5)$ & $100(30.9)$ & $91(28.1)$ \\
\hline & $45-59$ & $303(42.4)$ & $162(46.3)$ & $141(38.6)$ \\
\hline & 60plus & $386(52.5)$ & $197(51.8)$ & $189(53.2)$ \\
\hline \multirow[t]{4}{*}{ Baseline activity level } & Inactive & $520(41.1)$ & $283(44.0)$ & $237(38.0)$ \\
\hline & Moderately inactive & $125(37.9)$ & $64(40.0)$ & $61(35.9)$ \\
\hline & Moderately active & $158(46.2)$ & $66(41.0)$ & $92(50.8)$ \\
\hline & Active & $80(45.5)$ & $45(46.4)$ & $35(44.3)$ \\
\hline \multirow[t]{3}{*}{ Referral code } & CHD risk & $701(45.0)$ & $362(45.9)$ & $339(44.0)$ \\
\hline & Mental Health & $32(40.5)$ & $13(34.2)$ & $19(46.3)$ \\
\hline & Both & $173(33.1)$ & $91(36.0$ & $82(30.5)$ \\
\hline \multirow[t]{3}{*}{ WIMD tertile } & Low & $335(47.8)$ & $167(49.1)$ & $168(46.5)$ \\
\hline & Medium & $320(45.7)$ & $171(50.0)$ & 149 (41.6) \\
\hline & High & $228(32.6)$ & $118(31.9)$ & $110(33.3)$ \\
\hline \multirow[t]{2}{*}{ Trial status } & Control & $466(43.2)$ & & \\
\hline & Intervention & $440(40.7)$ & & \\
\hline \multirow[t]{3}{*}{ Adherence level } & Non-attendee & $28(17.4)$ & & \\
\hline & Partial completer & $143(32.1)$ & & \\
\hline & Completer & $269(56.9)$ & & \\
\hline
\end{tabular}

Table 2 Mean and SD values for psychosocial process variables in intervention and control groups, and odds ratios and $95 \% \mathrm{Cl}$ (unless otherwise indicated)

\begin{tabular}{|c|c|c|c|}
\hline Psychological process & Control & Intervention & Odds ratio $(95 \% \mathrm{Cl})$ \\
\hline Amotivation $(n=1098)$ & $0.46(0.76)$ & $0.33(0.66)$ & $0.69(0.54-0.90)$ \\
\hline External $(n=1102)$ & $0.67(0.88)$ & $0.68(0.85)$ & $1.07(0.86-1.34)$ \\
\hline Introjected $(\mathrm{n}=1102)$ & $1.66(1.14)$ & $1.94(1.19)$ & $1.56(1.25-1.95)$ \\
\hline Identified $(n=1105)$ & $2.50(1.03)$ & $2.83(1.91)$ & $1.66(1.33-2.07)$ \\
\hline Intrinsic $(n=1102)$ & $2.10(1.25)$ & $2.65(1.14)$ & $2.28(1.81-2.86)$ \\
\hline Autonomous motivation* $(n=1096)$ & $6.88(6.84)$ & $9.30(6.23)$ & $0.32(0.21$ to 0.44$)$ \\
\hline Self-efficacy $(n=1102)$ & $3.44(1.07)$ & $3.37(1.12)$ & 0.85 (0.68 to 1.05$)$ \\
\hline Social Support family (participation and involvement) $(n=1044)$ & $1.83(0.91)$ & $2.01(0.93)$ & $1.45(1.15-1.82)$ \\
\hline Family - exercise together ${ }^{\#}(n=1036)$ & $1.74(1.07)$ & $1.75(1.10)$ & $0.94(0.71-1.24)$ \\
\hline Family - verbal support ${ }^{\#}(n=1036)$ & $2.16(1.20)$ & $2.51(1.22)$ & $1.93(1.47-2.52)$ \\
\hline Family - planning exercise ${ }^{\#}(n=1040)$ & $1.52(0.90)$ & $1.64(1.00)$ & $1.42(1.06-1.90)$ \\
\hline Social support friends (participation and involvement) $(n=1006)$ & $0.84(0.85)$ & $0.98(0.84)$ & $1.37(1.09-1.73)$ \\
\hline Friends - exercise together ${ }^{\# \#}(n=1001)$ & $1.50(0.90)$ & $1.51(0.87)$ & $1.06(0.78-1.45)$ \\
\hline Friends - verbal support ${ }^{\# \#}(n=1004)$ & $1.71(0.98)$ & $1.89(1.06)$ & $1.37(1.04-1.81)$ \\
\hline Friends - planning exercise ${ }^{\# \#}(n=981)$ & $1.24(0.62)$ & $1.27(0.68)$ & $1.11(0.75-1.64)$ \\
\hline
\end{tabular}

*B-coefficients and 95\% Cls from linear regression.

\#Sub-scales emerging from factor analysis of 'family social support' scale.

\#"Sub-scales emerging from factor analysis of 'friends social support' scale.

significant associations $(p<0.05)$ in bold. 
Table 3 Percentage of participants with each level of activity at 12 months by trial arm, among patients referred for CHD risk factors, and who completed 6 month psychosocial process measures

\begin{tabular}{lllllll}
\hline \multicolumn{7}{c}{ Minutes of physical activity per week } \\
\cline { 2 - 7 } & $\mathbf{0}$ & $<\mathbf{1 5 0}$ & $\mathbf{1 5 0 - 2 9 9}$ & $\mathbf{3 0 0 - 5 9 9}$ & $\mathbf{6 0 0 +}$ & \\
\hline Control & $56(15.5)$ & $108(29.8)$ & $83(22.9)$ & $78(21.6)$ & $37(10.2)$ & 362 \\
Intervention & $38(11.2)$ & $88(26.0)$ & $81(23.9)$ & $82(24.2)$ & $50(14.8)$ & 339 \\
\hline
\end{tabular}

of patients who were already active at baseline. There were no significant interaction effects, except for baseline physical activity level (with already active patients significantly less likely to report improved motivation).

\section{Discussion}

In summary, this paper indicates that: i) referral to NERS led to improvements in autonomous motivation and social support from family and friends, although no effects were found upon self-efficacy, ii) change in autonomous motivation partially mediated change in 12 month physical activity, with change in autonomous motivation explaining $44 \%$ of the total intervention effect on physical activity, though there was less clear evidence of mediation by change in social support and iii) there was no evidence of socio-demographic patterning in changes in autonomous motivation, other than a greater effect on motivation among patients who were not already active at baseline.

Improvements in autonomous motivation after attendance at an exercise referral scheme have been described by a number of previous studies (e.g. [21]), and are consistent with the NERS logic model [13], which highlighted elicitation and strengthening of internal motivation as a key mechanism of the intervention. To our knowledge, no previous studies of exercise on referral have assessed change in autonomous motivation within a randomised controlled design and assessed the degree to which this change can explain longer term change in physical activity. However, in a study of a weight loss intervention with 258 female participants aged 25-50 years, post intervention need support and autonomous motivation were found to partially mediate the level of moderate to vigorous exercise one year post intervention and weight loss two years post intervention [41].

Notably, within NERS, improvement in autonomous motivation occurred despite weak delivery of some core components specifically intended to enhance internal motivation, such as motivational interviewing, goal setting and relapse prevention consultations [13]. Hence, as reported in qualitative interviews $[13,42]$ improvements in autonomous motivation most likely arose in large part from the support of professionals during the exercise programme, and from the provision of a supportive network of patients who provide empathy and realistic social comparisons [13,42]. Whether effects on autonomous motivation could be enhanced further through more effective delivery of strategies such as motivational interviewing and goal setting remains an empirical question for future research.

The lack of effect on self-efficacy appears somewhat at odds with earlier qualitative data. However, it is possible that the self-efficacy questionnaire, validated with younger samples in the US in the 1980s and focused upon confidence to overcome barriers, failed to sufficiently capture change in the specific dimensions of self-efficacy of greatest relevance to participants. Participant interviews emphasised issues such as increased confidence in using machinery, or in their ability to exercise safely while avoiding exacerbation of injury or illness [13,42]. A further reason for this lack of effect could be that participants may have had inflated initial efficacy expectations at baseline. These may have been reduced in the intervention group due to the introduction of new barriers in terms of time and finances, whereas they may have been maintained in the control group. A ceiling effect may have occurred, whereby only participants with the highest levels of self-efficacy may have adhered to the trial long enough to provide data at each time point. It is also possible that impacts on self-efficacy could be improved through more effective delivery of goal setting. As reported elsewhere, goal setting within NERS commonly centred around vague and unmeasurable, or in some cases unrealistic goals. The lack of clear measurable goals may have made it difficult to review and feedback progress to reinforce self-efficacy, while failure to achieve unrealistic goals may have led to reduced self-efficacy for some.

Table 4 Mediation of change in physical activity by psychological process variables (OR and $95 \%$ Cls from ordinal regression models)

\begin{tabular}{|c|c|c|c|c|}
\hline & \multirow{2}{*}{$\begin{array}{l}\text { Model } 1 \\
\text { Intervention } \rightarrow \text { Physical } \\
\text { activity (unadjusted) }\end{array}$} & \multicolumn{2}{|l|}{ Model 2} & \multirow{2}{*}{$\begin{array}{l}\text { Pseudo } R^{2} \\
\text { for model } 2\end{array}$} \\
\hline & & $\begin{array}{l}\text { Mediator } \rightarrow \text { Physical activity } \\
\text { (where entered alongside trial term) }\end{array}$ & $\begin{array}{l}\text { Intervention } \rightarrow \text { Physical activity } \\
\text { after adjusting for mediator }\end{array}$ & \\
\hline $\begin{array}{l}\text { Autonomous motivation } \\
(n=663)\end{array}$ & 1.39 (1.06 to 1.83$)$ & 1.36 (1.17 to 1.58$)$ & 1.27 (0.96 to 1.67$)$ & 0.03 \\
\hline SS family (Sallis) $(n=633)$ & $1.46(1.10$ to 1.93$)$ & $1.29(1.11$ to 1.51$)$ & 1.38 (1.04 to 1.84$)$ & 0.03 \\
\hline SS friends $(n=611)$ & 1.40 (1.05 to 1.87$)$ & 1.30 (1.06 to 1.59$)$ & 1.37 (1.03 to 1.83$)$ & 0.03 \\
\hline
\end{tabular}

Significant associations $(p<0.05)$ in bold. 
Table 5 Moderators of change in autonomous motivation for exercise (B-coefficients and 95\% Cls from linear regression models)

Relative Autonomy Index (RAI) intervention effect in subgroups

\begin{tabular}{|c|c|c|c|c|c|c|}
\hline & & $\begin{array}{l}\text { Gender (subgroup } \\
1=\text { female } N=680, \\
2=\text { male } N=364 \text { ) }\end{array}$ & $\begin{array}{l}\text { Reason for referral } \\
(1=\text { CHD N }=783 \\
2=\text { Mental health } \\
N=261)\end{array}$ & $\begin{array}{l}\text { Age }(1=16-44 N=246 \\
2=45-59 N=362 \\
3=60+N=421)\end{array}$ & $\begin{array}{l}\text { Deprivation }(1=\text { low } \\
N=3712=\text { medium } \\
N=3713=\text { high } \\
N=272)\end{array}$ & $\begin{array}{l}\text { Baseline PA }(1=\text { inactive } \\
N=6222=\text { moderately } \\
\text { inactive } N=150 \\
3=\text { active } N=263 \text { ) }\end{array}$ \\
\hline \multirow{2}{*}{\multicolumn{2}{|c|}{ Subgroup 1}} & 0.31 & 0.33 & 0.36 & 0.24 & 0.40 \\
\hline & & (0.17 to 0.46$)$ & $(0.20$ to 0.46$)$ & (0.12 to 0.59$)$ & $(0.05$ to 0.43$)$ & $(0.26$ to 0.54$)$ \\
\hline \multirow{2}{*}{\multicolumn{2}{|c|}{ Subgroup 2}} & 0.31 & 0.29 & 0.32 & 0.36 & 0.46 \\
\hline & & (0.11 to 0.50$)$ & (0.06 to 0.52$)$ & $(0.12$ to 0.51$)$ & $(0.16$ to 0.55$)$ & $(0.15$ to 0.76$)$ \\
\hline \multirow{2}{*}{\multicolumn{2}{|c|}{ Subgroup 3}} & & & 0.33 & 0.39 & 0.07 \\
\hline & & & & (0.15 to 0.51$)$ & (0.17 to 0.62$)$ & $(-0.17$ to 0.31$)$ \\
\hline \multicolumn{7}{|c|}{ Interaction effects (whole sample) } \\
\hline & & $N=1096$ & $N=1096$ & $N=1096$ & $N=1063$ & $N=1096$ \\
\hline \multirow{2}{*}{\multicolumn{2}{|c|}{ Trial effects }} & 0.31 & 0.33 & 0.34 & 0.24 & 0.40 \\
\hline & & $(-0.03$ to 0.65$)$ & $(0.20$ to 0.46$)$ & $(0.10$ to 0.58$)$ & (0.05 to 0.43$)$ & $(0.26$ to 0.55$)$ \\
\hline \multirow[t]{4}{*}{ Moderator } & Subgroup 2vs1 & 0.04 & 0.05 & 0.25 & -0.13 & 0.21 \\
\hline & & $(-0.13$ to 0.21$)$ & $(-0.14$ to 0.24$)$ & (0.04 to 0.47$)$ & $(-0.44$ to 0.06$)$ & $(-0.03$ to 0.45$)$ \\
\hline & Subgroup 3vs1 & & & 0.57 & 0.01 & 0.57 \\
\hline & & & & (0.36 to 0.77$)$ & $(-0.19$ to 0.22$)$ & (0.38 to 0.77 ) \\
\hline \multirow{4}{*}{$\begin{array}{l}\text { Interaction } \\
\text { effects }\end{array}$} & Subgroup 2vs 1 & 0.01 & -0.03 & -0.03 & 0.13 & 0.00 \\
\hline & & $(-0.23$ to 0.25$)$ & $(-0.29$ to 0.23$)$ & $(-0.33$ to 0.28$)$ & $(-0.14$ to 0.41$)$ & $(-0.33$ to 0.33$)$ \\
\hline & Subgroup 3vs1 & & & -0.01 & 0.13 & -0.31 \\
\hline & & & & $(-0.30$ to 0.29$)$ & ( -0.15 to 0.42$)$ & $(-0.58$ to -0.05$)$ \\
\hline
\end{tabular}

Significant associations $(\mathrm{p}<0.05)$ in bold.

Previous studies of physical activity interventions have shown social support from family and friends to be associated with the uptake and maintenance of physical activity $[4,43,44]$. While no baseline measure of social support from friends and family was available, and hence it was not possible to ascertain the extent to which baseline levels of support moderated patient responses to the intervention, this paper indicates that perceived support from friends and families increased as a result of referral to NERS. It is possible that identification of a patient as 'at-risk' by a health professional encouraged their friends and family to increase their support for change efforts. In qualitative interviews, some patients described how referral to the scheme legitimised their taking time out from family responsibilities, giving them a set time and place to act on GP advice to take up exercise and leading family to support their taking time out [13].

However, while autonomous motivation emerged as a mediator of long-term change in 12 month physical activity, there was less clear evidence of mediation by social support variables. While a near significant indirect effect was observed for family social support, there was no evidence of mediation by social support from friends. Hence, it is possible either that increased social support was not sufficient to translate into increased physical activity, or that increases in social support observed at 6 months returned to baseline levels by 12 month followup. Indeed, within qualitative interviews, some commented that the support from their families triggered by referral to NERS would not last once they were no longer in a set programme prescribed by a health professional. Notably, as with self-efficacy, qualitative interviews focused on aspects of social support which were not captured within the measures adopted for the trial, emphasising the support of the professional and the emergence of social support from other patients. Hence, it remains plausible that effects of the intervention were mediated by alternative aspects of social support.

No evidence was found that change in autonomous motivation was moderated by demographic factors, such as age, gender or socioeconomic status. Hence emergence of patterning in adherence reported elsewhere [13] or effects on physical activity [9], appear to have arisen from processes not measured by this study. Notably however, there was a tendency for greatest improvements in autonomous motivation among those patients who were doing least exercise at baseline. This runs somewhat contrary to data from exercise professionals $[13,42]$ which suggested that the scheme most effectively engaged individuals who were already highly motivated 
toward exercise, and indicates that despite the aforementioned limited delivery of some components specifically intended to enhance internal motivation (e.g. motivational interviewing; [13]), the intervention did effectively enhance motivation among previously inactive patients.

\section{Strengths and limitations}

To our knowledge, this study is the first to examine short-term impacts of an exercise referral scheme on psychosocial processes within a rigorous randomised trial design, and to link changes in psychosocial processes to longer term physical activity. The nature of the study as a pragmatic policy trial means that the findings have good external validity, and are directly reflective of real world practice. Nevertheless, there are some limitations which should be considered. The study is limited somewhat by attrition, and in particular, by the fact that the likelihood of providing complete data was patterned by factors which also predicted adherence to the intervention, such as age and mental health status. This may have led to overestimation of effects on autonomous motivation (that is, those whose motivation did not improve through participating in NERS, may have simply dropped out of the trial). Collection of baseline measure of psychosocial process variables may have provided greater assurance that these did not differ at baseline, though would also have enhanced respondent burden, risking biasing recruitment and retention further. Our analysis approach of dividing key outcomes into categories to overcome substantial skewness was selected to ensure comparability with the main trial analysis, through replicating and extending it. Alternative approaches include generalized linear models, which may provide more power, though depend on stricter distributional assumptions. In addition, the study would ideally have had a longer lead in period for modelling and theory development $[45,46]$, which was not possible given that it was a pragmatic trial of a policy initiative. Such a lead in phase would for example have facilitated the collection of qualitative data on psychosocial processes, and development and validation of bespoke measures which captured the specific aspects of self-efficacy and social support cited within patient interviews as important in promoting behavioural change.

\section{Conclusions}

This study offers important insights into and practical implications for the functioning of exercise referral schemes, due to the use of a pragmatic policy trial design, which involved the evaluation of a real-world intervention, whilst maintaining high standards of scientific rigour. Such evaluations are essential in order to bridge the gap between academia and policy makers by providing evidence of the highest possible relevance to real world practice [47]. The effects of an exercise referral scheme on physical activity explained via improvements in autonomous motivation provides support for recent efforts to explicitly base the design of exercise referral schemes on self-determination theory [48], and emphasis on enhancing baseline motivation within Department of Health [7] guidance for exercise referral. Future evaluations should consider designing and validating bespoke measures of psychosocial processes, such as self-efficacy, which capture the aspects of those processes most likely to be affected by exercise referral. In addition, while measures included emphasised social support from friends and family, development and testing of measures to capture more emergent aspects of social support, such as that from the professional or other patients should be considered in future evaluations. Studies should also consider whether, as suggested by qualitative data reported elsewhere, these emerging forms of social support predict long term maintenance of behavioural change. More research is needed to explain socio-demographic patterning in responses to ERS, with changes in motivation occurring among almost all sub-groups of participants, though not always leading to higher adherence or behavioural change. This perhaps highlights the importance of socio-ecological approaches to developing and evaluating behaviour change interventions, which view individual level factors such as motivation as important, yet emphasise the need to understand how influences beyond the individual (e.g. organisational, community or policy-level factors), lead to patterning in responses to individual-level interventions.

\section{Competing interests}

The authors declare that they have no competing interests.

\section{Authors' contributions}

$\mathrm{HL}$ and GM conducted the analysis and wrote the paper. SM led the trial from which data were drawn. All authors provided comments on drafts and approved the final manuscript.

\section{Acknowledgements}

The NERS trial was funded by the Welsh Government Health Improvement division. The authors thank the NERS evaluation team, the scheme's coordinators (Elaine McNish and Jeannie Wyatt-Williams), and Janine Hale and Chris Roberts within the Welsh Government for providing information and support to facilitate the study. The work was supported by Centre for the Development and Evaluation of Complex Interventions for Public Health Improvement (DECIPHer), a UKCRC Public Health Research: Centre of Excellence. Funding from the British Heart Foundation, Cancer Research UK, Economic and Social Research Council (RES-590-28-0005), Medical Research Council, the Welsh Assembly Government and the Wellcome Trust (WT087640MA), under the auspices of the UK Clinical Research Collaboration, is gratefully acknowledged. We are also grateful to Mark Kelson for advice regarding aspects of the statistical analysis.

\section{Author details}

'DECIPHer, School of Social Sciences, Cardiff University, 1-3 Museum Place, Cardiff, Wales, UK. ${ }^{2} \mathrm{MRC} / \mathrm{CSO}$ Social \& Public Health Sciences Unit (SPHSU), University of Glasgow, Glasgow, Scotland, UK.

Received: 14 November 2013 Accepted: 18 August 2014

Published online: 27 August 2014 
References

1. Chief Medical Officer: At least five a week: evidence on the impact of physical activity and its relationship to health. London: Department of Health; 2004.

2. NICE: Four commonly used methods to increase physical activity: brief interventions in primary care, exercise referral schemes, pedometers and community-based exercise programmes for walking and cycling. In Public health intervention guidance No 2. London: NICE; 2006.

3. Sowden $S L$, Raine R: Running along parallel lines: how political reality impedes the evaluation of public health interventions. A case study of exercise referral schemes in England. J Epidemiol Community Health 2008 , 62(9):835-841.

4. Williams NH, Hendry M, France B, Lewis R, Wilkinson C: Effectiveness of exercise-referral schemes to promote physical activity in adults: systematic review. Br J Gen Pract 2007, 57:979-986.

5. Morgan O: Approaches to increase physical activity: reviewing the evidence for exercise-referral schemes. Public Health 2005, 119(5):361-370.

6. Pavey TG, Taylor AH, Fox KR, Hillsdon M, Anokye N, Campbell JL, Foster C, Green C, Moxham T, Mutrie N, Searle J, Trueman P, Taylor RS: Effect of exercise referral schemes in primary care on physical activity and improving health outcomes: systematic review and meta-analysis. BMJ 2011, 343:d6462.

7. Department of Health: National quality assurance framework for exercise referral schemes. London: The Stationery Office; 2001.

8. Murphy S, Raisanen L, Moore G, Edwards R, Linck P, Williams N, Ud Din N, Hale J, Roberts C, McNaish E, Moore L: A pragmatic randomised controlled trial of the welsh national exercise referral scheme: protocol for trial and integrated economic and process evaluation. BMC Public Health 2010, 10(1):352.

9. Murphy SM, Edwards RT, Williams N, Raisanen L, Moore G, Linck P, Hounsome N, Din NU, Moore L: An evaluation of the effectiveness and cost effectiveness of the national exercise referral scheme in wales, UK: a randomised controlled trial of a public health policy initiative. J Epidemiol Community Health 2012, 66:745-753.

10. Moore G, Audrey S, Barker M, Bond L, Bonell C, Cooper C, Hardeman W, Moore L, O'Cathain A, Tinati T, Wight D, Baird J: Process evaluation in complex public health intervention studies: the need for guidance. J Epidemiol Community Health 2014, 68(2):101-102.

11. Pawson R, Tilley N: Realistic evaluation. London: Sage; 1997.

12. Moore GF, Moore L, Murphy S: Integration of motivational interviewing into practice in the national exercise referral scheme in wales: a mixed methods study. Behav Cogn Psychother 2011, 40(3):313-330. FirstView.

13. Moore GF, Raisanen L, Moore L, Din NU, Murphy S: Mixed-method process evaluation of the welsh national exercise referral scheme. Health Educ 2013, 113(6):476-501.

14. Bonell C, Fletcher A, Morton M, Lorenc T, Moore L: Realist randomised controlled trials: a new approach to evaluating complex public health interventions. Soc Sci Med 2012, 75(12):2299-2306

15. Bandura A: Self-efficacy: toward a unifying theory of behavioral change. Psychol Rev 1977, 84(2):191.

16. Deci EL, Ryan RM: Intrinsic motivation and self determination in human behaviour. New York: Plenum; 1985.

17. Fortier MS, Duda JL, Guerin E, Teixeira PJ: Promoting physical activity: development and testing of self-determination theory-based interventions. Int I Behav Nutr Phys Act 2012, 9(1):20.

18. Teixeira PJ, Carraça EV, Markland D, Silva MN, Ryan RM: Exercise, physical activity, and self-determination theory: a systematic review. Int J Behav Nutr Phys Act 2012, 9(1):78.

19. Edmunds J, Ntoumanis N, Duda JL: Adherence and well-being in overweight and obese patients referred to an exercise on prescription scheme: a self-determination theory perspective. Psychol Sport Exerc 2007, 8(5):722-740.

20. Morton KL, Biddle SJH, Beauchamp MR: Changes in self-determination during an exercise referral scheme. Public Health 2008, 122(11):1257-1260.

21. Markland D, Tobin VJ: Need support and behavioural regulations for exercise among exercise referral scheme clients: the mediating role of psychological need satisfaction. Psychol Sport Exerc 2010, 11(2):91-99.

22. Williams G: Improving patients' health through supporting the autonomy of patients and providers. In Handbook of self-determination research. Edited by Deci E, Ryan R. Rochester, NY: Rochester Press; 2002:233-254.
23. Anderson ES, Wojcik JR, Winett RA, Williams DM: Social-cognitive determinants of physical activity: the influence of social support, self-efficacy, outcome expectations, and self-regulation among participants in a church-based health promotion study. Health Psychol 2006, 25(4):510.

24. Jones F, Harris P, Waller $\mathrm{H}$, Coggins A: Adherence to an exercise prescription scheme: the role of expectations, self-efficacy, stage of change and psychological well-being. Br J Health Psychol 2005, 10(3):359-378

25. Sniehotta FF, Scholz U, Schwarzer R: Bridging the intention-behaviour gap: planning, self-efficacy, and action control in the adoption and maintenance of physical exercise. Psychol Health 2005, 20(2):143-160

26. Carron AV, Hausenblas HA, Mack D: Social influence and exercise: a meta-analysis. J Sport Exerc Psychol 1996, 18:1-16.

27. NICE: Rapid review of the economic evidence of physical activity interventions. National Institute for Health and Clinical Excellence; 2006.

28. Sarkin J, Campbell J, Gross L, Roby J, Bazzo S, Sallis J, Calfas K: Project GRAD seven day physical activity recall interviewer's manual. Med Sci Sports Exerc 1997, 29(6):S91-S102

29. Markland $\mathrm{D}$, Tobin $\mathrm{V}$ : A modification to the behavioural regulation in exercise questionnaire to include an assessment of amotivation. J Sport Exerc Psychol 2004, 26(2):191-196.

30. Sallis JF, Pinski RB, Grossman RM, Patterson TL, Nader PR: The development of self-efficacy scales for healthrelated diet and exercise behaviors. Health Educ Res 1988, 3(3):283-292.

31. Sallis JF, Grossman RM, Pinski RB, Patterson TL, Nader PR: The development of scales to measure social support for diet and exercise behaviors. Prev Med 1987, 16(6):825-836.

32. Blair SN, Haskell WL, Ho P, Paffenbarger RS, Vranizan KM, Farquhar JW, Wood PD: Assessment of habitual physical activity by a seven-day recall in a community survey and controlled experiments. Am J Epidemiol 1985, 122(5):794-804.

33. Donnelly JE, Jacobsen DJ, Jakicic JM, Whatley JE: Very low calorie diet with concurrent versus delayed and sequential exercise. Int J Obes 1994, 18(7):469-475.

34. Dunn AL, Marcus BH, Kampert JB, Garcia ME, Kohl HW 3rd, Blair SN: Comparison of lifestyle and structured interventions to increase physical activity and cardiorespiratory fitness: a randomized trial. [see comment]. Jama 1999, 281(4):327-334.

35. Lamb SE, Bartlett HP, Ashley A, Bird W: Can lay-led walking programmes increase physical activity in middle aged adults? A randomised controlled trial. J Epidemiol Community Health 2002, 56(4):246-252.

36. Harrison RA, Roberts C, Elton PJ, Harrison RA, Roberts C, Elton PJ: Does primary care referral to an exercise programme increase physical activity one year later? A randomized controlled trial. J Public Health (Oxf) 2005, 27(1):25-32.

37. Stevens W, Hillsdon M, Thorogood M, McArdle D, Eaton CB: Cost-effectiveness of a primary care based physical activity intervention in 45-74 year old men and women: A randomised controlled trial. Br J Sports Med 1998, 32(3):236-241.

38. Taylor AH, Doust J, Webborn N: Randomised controlled trial to examine the effects of a GP exercise referral programme in Hailsham, East Sussex on modifiable coronary heart disease risk factors. J Epidemiol Community Health 1998, 52(9):595-601.

39. Baron RM, Kenny DA: The moderator-mediator variable distinction in social psychological research: conceptual, strategic, and statistical considerations. J Pers Soc Psychol 1986, 51(6):1173.

40. Mackinnon DP, Dwyer JH: Estimating mediated effects in prevention studies. Eval Rev 1993, 17(2):144-158.

41. Silva MN, Markland D, Carraça EV, Vieira PN, Coutinho SR, Minderico CS, Matos MG, Sardinha LB, Teixeira PJ: Exercise autonomous motivation predicts 3-yr weight loss in women. Med Sci Sports Exerc 2011, 43(4):728-737.

42. Moore G, Moore L, Murphy S: Facilitating adherence to physical activity: exercise professionals' experiences of the national exercise referral scheme in wales. A qualitative study. BMC Public Health 2011, 11(1):935

43. Sallis J, Owen N: Physical activity and behavioral medicine. Behavioral medicine and health psychology, Volume 3. SAGE: Thousand Oaks, CA; 1998.

44. Allender S, Cowburn G, Foster C: Understanding participation in sport and physical activity among children and adults: a review of qualitative studies. Health Educ Res 2006, 21(6):826-835. 
45. Craig P, Dieppe P, Macintyre S, Michie S, Nazareth I, Petticrew M: Developing and evaluating complex interventions: new guidance. Medical Research Council; 2008.

46. Craig P, Dieppe P, Macintyre S, Michie S, Nazareth I, Petticrew M:

Developing and evaluating complex interventions: the new medical research council guidance. BMJ 2008, 337:a1655.

47. Maclure M: Explaining pragmatic trials to pragmatic policy-makers. Can Med Assoc J 2009, 180(10):1001-1003.

48. Jolly K, Duda J, Daley A, Eves F, Mutrie N, Ntoumanis N, Rouse P, Lodhia R, Williams G: Evaluation of a standard provision versus an autonomy promotive exercise referral programme: rationale and study design. BMC Public Health 2009, 9(1):176.

doi:10.1186/s12966-014-0109-9

Cite this article as: Littlecott et al:: Psychosocial mediators of change in physical activity in the Welsh national exercise referral scheme:

secondary analysis of a randomised controlled trial. International Journal of Behavioral Nutrition and Physical Activity 2014 11:109.

\section{Submit your next manuscript to BioMed Central and take full advantage of:}

- Convenient online submission

- Thorough peer review

- No space constraints or color figure charges

- Immediate publication on acceptance

- Inclusion in PubMed, CAS, Scopus and Google Scholar

- Research which is freely available for redistribution 